\title{
MASSIVE COLLAPSE OF THE LUNG AS A RESULT OF GUNSHOT WOUNDS, WITH ESPECIAL REFERENCE TO WOUNDS OF THE CHEST
}

\author{
BY JOHN ROSE BRADFORD
}

With Plates 10-12

\section{Introduction.}

THE study of the physical signs present in the chest in cases of gunshot wounds involving the chest-wall or its contents has been a subject of great interest during the present campaign, owing to the variety, complexity, and difficulty of satisfactory interpretation of the signs observed in many cases. On a priori grounds, it might be thought, inasmuch as the more common effect of such injuries is to cause either a collection of bloody fluid or a mixture of such fluid with air or gas in the pleural cavity, that the physical signs would be merely those generally regarded as characteristic of the presence of fluid or gas or both in the pleural cavity. A very slight experience of chest wounds, even when the lesion produced is a simple, sterile haemothorax, shows that this is far from the truth, and that in a very large number of cases, perhaps even in all, the signs are really different to those hitherto regarded as characteristic of the presence of fluid or gas in the pleura. Some observers have sought to explain the discrepancies by the suggestion that the lesions often present in the lung as the direct result of the laceration, \&c., produced by the missile are the cause of the complexity and diversity of the signs present. Doubtless there is much truth in this view, but the object of the present paper is to draw attention more especially to another and relatively little-known condition, i. e. massive collapse of the lung, as a most important factor in the production of the clinical phenomena seen in cases of gunshot injuries of the chest. In the opinion of the writer, this is not only a most characteristic but also a common result in cases of gunshot wounds of the chest. It was not fully recognized until after a very extensive experience of chest cases, although it is, in all probability, really of frequent occurrence. It may be of interest to record shortly the stages in the process of its recognition.

1. The diaphragm in cases of simple sterile haemothorax was found, on clinical examination, to be abnormally high. This fact is most readily demon[Q. J. M., Oct., Ig18, and Jan., 1919.] 
strated in cases of left-sided haemothorax, since it is then easy to detect the upward displacement of the stomach and colon by ordinary percussion. X-ray examination not only confirmed the view that the diaphragm was displaced, but also showed that it was immobile on the injured side.

2. More extended experience and more careful observation showed that in many cases of haemothorax, and especially in cases of sterile haemothorax, the affected side of the chest was not only immobile, but the chest-wall was actually retracted, notwithstanding the presence of a considerable quantity of fluid in the pleural cavity. In some of these cases displacement of the heart towards the opposite or uninjured side was also present. Retraction of the chest-wall, notwithstanding the presence of fluid in the pleural cavity, is a common phenomenon in haemothorax; it is not always accompanied by this displacement of the heart, but when this is the case the clinical picture is a very remarkable one, and strikingly different to that associated with the mere presence of fluid, e.g. pleural effusion, in the chest. It is, of course, well known that in certain chronic pleural effusions, where the lung has been long compressed and has undergone secondary changes, such a condition may exist; but in haemothorax this elinical picture is not only an early one, but quite often is transitory, the retraction of the chestwall disappearing and being perhaps replaced by bulging. This is especially seen in cases where the haemothorax is infected and there is a rapid increase in the amount of pleural exudate.

3. A small number of cases showed physical signs indicative of the presence of collapse, involving the whole of the lung on the side of the haemothorax, although the haemothorax was quite small in amount, in fact often so small as not to require special treatment. Such cases might, for instance, show dullness on percussion up to the clavicle, and yet the amount of fluid in the chest was not more than a few ounces; in other words, the degree of collapse was quite out of proportion to the size of the haemothorax.

4. At this stage in the study of haemothorax, the fact that the collapse of the lung should not necessarily be regarded as solely due to the bloody effusion was being slowly appreciated, and then a series of cases was observed where massive collapse involving the entire lung occurred on the side opposite to that wounded, and not only as a result of unilateral wounds limited to the chest-wall, but often of wounds slight or even trivial in character.

5. The recognition of massive contralateral collapse' as a result of contour wounds led to the detection of less extensive areas of collapse as one of the contralateral complications of haemothorax, whereas previously cases presenting similar physical signs had been regarded as probably pneumonic in character. Although pneumonia may occur as a contralateral complication of haemothorax, especially in infected cases, yet many cases regarded as pneumonic are really not so, and the complication present is collapse and not pneumonic consolidation. The fact that contralateral massive collapse may occur as a result of a nonpenetrating wound of the thoracic parietes, and the occurrence of homolateral massive collapse, involving the entire lung, in cases of slight haemothorax, raises 
the question whether the collapse present in ordinary haemothorax is always due solely to the effusion, or whether it is not really an associated phenomenon of independent and different origin. If the collapse of the lung associated with haemothorax is not solely dependent upon the mere presence of the effused blood in the pleura, but is really produced in some other way, this might serve to explain the discrepancies between the clinical phenomenon as seen in cases of haemothorax and those present in pleural effusion. It seems obvious to regard the ordinary collapse of the lung in haemothorax as due to the mere effusion of blood, but if this really be the case, it is very difficult to understand why the clinical phenomena are so often different to those of other forms of pleural effusion. The raised position of the diaphragm, with the accompanying increase in the resonant area known as Traube's space, in haemothorax, instead of the replacement of this resonant area by dullness, as in pleural effusion, is more especially difficult to explain. At first the view was entertained that this difference might be due to the following considerations. The gunshot wound of the chest might cause the entry of air jnto the pleural cavity and so lead, even in a case of simple haemothorax, to the production of an initial pneumothorax of transitory duration; the blood might be poured out with the lung already collapsed, and then, the air being absorbed, the case would ultimately become one of simple haemothorax. The occurrence of contralateral massive collapse after wounds limited to the parietes, and where there was no penetration, showed that most extensive collapse could occur as a result of non-penetrating wounds, and therefore it is not necessary to invoke the hypothesis of transitory pneumothorax in order to explain the presence of collapse in haemothorax.

The writer is inclined to regard collapse of the lung in gunshot wounds of the chest as a phenomenon due directly to the injury of the chest, and although often accompanied by haemothorax, yet when so associated as not necessarily due solely to the latter. The most easily recognized cases of massive collapse are those associated with non-penetrating wounds of the chest-wall, and more especially those where the collapse occurs on the side opposite to that injured, and where it involves the entire lung. In such cases the diagnosis is easy, as the clinical picture is very definite, but other forms are by no means so easy to recognize, since if they are of the contralateral variety the area involved may be small, and the signs resemble those of pneumonia ; and if they are of the homolateral type, other difficulties arise. Thus the homolateral variety is often associated with haemothorax, and much difficulty may be experienced in differentiating the physical signs of collapse from those due to other causes, such as fluid in the pleura, or consolidation of the lung, due to haemorrhagic infiltration of the lung produced, either directly by the missile lodged in or perforating the lung, or indirectly, merely as a result of the impact of the bullet on the chest-wall. Further, in the contralateral variety, when this is associated with haemothorax on the injured side, there may be difficulty in interpreting the significance to be attached to displacement of the heart, whether it is mainly due to the haemothorax or whether it is due to the supposed contralateral collapse. For these 
reasons it is important to recognize that the typical examples of massive collapse in gunshot injuries of the chest should be sought for in cases of non-penetrating unilateral wounds of the chest-wall, and on the side opposite to that wounded; but massive collapse is by no means limited to such cases.

In civil practice massive collapse of the lung has been described by several observers, and more especially by Pasteur, ${ }^{1}$ as occurring in a number of conditions ; the best-known variety is that seen after certain abdominal operations and injuries. Pasteur has also described it in cases of death from diphtheritic paralysis, and in these cases it has been thought to owe its origin to paralysis of the diaphragm. Although the condition described in the present communication has great analogies with that described in civil practice, it is perhaps not certain that it is identical with it. In certain diseases, especially enteric fever and pneumonia, extensive collapse of the lungs is sometimes seen, and this may sometimes be not only lobar in its distribution, but also unilateral, so that it greatly resembles massive collapse, or is indistinguishable from it. More usually, however, it is not strictly lobar and involves more especially the posterior rounded portion of the lung that occupies the most dependent part of the chest in the recumbent posture. Such cases are well known and are quite different from those here described. Only one case of massive collapse, involving the whole of one lung, had fallen under the observation of the writer prior to the War, and in this case the cause was simple, i. e. the impaction of an acorn in the left bronchus. The expulsion of the foreign body by a violent fit of coughing was followed by a complete recovery.

It is difficult to form an opinion as to the frequency of occurrence of massive collapse. In civil practice, if the cases occurring after abdominal operations be excluded, it would seem to be extremely rare unless there is some obvious lesion interfering with the expansion of the lung, e.g. palsy of the diaphragm, foreign body in the bronchus, \&c., and, as mentioned above, no case has ever fallen under the observation of the writer during a long hospital experience.

During the present campaign, on the other hand, a considerable number of cases have been seen; but here, also, it is not possible to give figures as to the frequency of its incidence, owing to the outstanding fact that it is so often not recognized unless specially sought for, so many of the patients do not present any urgent symptoms, and if symptoms are present the signs are often wrongly attributed to pneumonia. Most of the cases on which this paper is founded have been patients suffering from gunshot wounds of the chest, but typical cases of extensive massive collapse have been seen as a result of other wounds, more especially wounds of the buttock and wounds of the pelvis. In a few cases it has occurred as a complication of wounds of the thigh. No case is included where the wound was one of the abdomen. No opportunity was afforded the writer to see any large series of abdominal wounds; and further, such cases are not suitable for the study of a chest condition like collapse. For these reasons

2 Pasteur, Brit. Joum. of Surgery, i, No. 4, 1914. 
the present communication contains no facts as to massive collapse in abdominal gunshot wounds.

The term 'collapse of the lung', unless qualified, is used in this paper in the sense of a collapse of the lung more or less extensive, where there is no gross lesion such as fluid or air in the pleura to account for its presence. The collapse present in association with haemothorax is not fully dealt with, except in the cases of massive collapse of the entire lung in association with a small haemothorax. In the study of contralateral collapse it is essential to consider especially cases of unilateral gunshot wounds of the chest, and even then much care must be taken to determine whether a bilateral chest lesion has not really been produced, or even a contralateral one, as sometimes a bullet will pass through one side of the chest without producing any appreciable lesion on that side, and yet produce serious lesions on the opposite side. These difficulties, however, are not present in cases of unilateral non-penetrating wounds of the chest-wall.

\section{Varieties of Massive Collapse.}

Collapse may involve only a portion of one lobe of the lung, or an entire lobe, or even one entire lung. In cases of unilateral gunshot wounds of the chest, it may be present either on the injured side only, or on the opposite side only, or on both sides. It may apparently vary in the degree of its development, as well as in the extent of lung involved; but this is often difficult to determine witb any degree of accuracy, since only comparatively rough clinical methods are available for this purpose. At any rate, homolateral, contralateral, and bilateral collapse may be recognized, and it may be partial, lobar, or total, meaning by total a condition where the whole of one lung is involved. In some cases the condition seems to increase whilst the patient is under observation; in other words, it is progressive, involving first a small portion and then later a larger area of the lung. This, however, is rare, and certainly, in the cases where the whole of one lung has been affected, the entire lung has usually been involved when the patient was first examined and the condition recognized.

In the slighter forms, where the collapse is at first limited to a portion of one lobe, it is not very unusual for the area of lung involved to increase in size and so cause a considerable increase in the area in which physical signs are present. There is another form of variation that may occur in all varieties of massive collapse; in this the area involved does not increase in extent, but the affected lung becomes either less or more airless. The physical signs present are not found, in these cases, to extend and involve a larger area of lung, but they alter in character whilst remaining limited to their original area. Thus, at a first examination, the only abnormal signs may be weakness of the breath-sounds over a certain region, and were it not for the presence of other signs, and especially displacement of the heart, the correct interpretation of the weakened breath-sounds might not be arrived at. Then, after the lapse of some hours, these signs are replaced by others, such as loud tubular or even amphoric breath- 
Ing, showing that certain bronchial tubes are now pervious, and that the respiratory movements are adequate to cause the entry of air into the lung. In other instances the reverse change takes place, and weakened breath-sounds are only heard over an area where previously loud tubular breathing had been present. These variations in the degree of collapse are probably of mixed origin, sometimes depending on varying degrees of bronchial obstruction, and perhaps in other cases dependent upon a return of efficient respiratory movements.

Massive collapse involving the whole of one lung is the form that is most easily recognized clinically, inasmuch as the physical signs of this condition are extremely well marked. Two varieties have been met with, both associated with unilateral wounds of the chest. In the first, or homolateral form, the massive total collapse is on the same side as the injury, and in cases of perforating wounds may be accompanied with a small haemothorax. In the second, or contralateral variety, the collapse is on the side of the chest opposite to that wounded, and the wound in all these cases has been a non-penetrating one, limited to the parietes, and not causing, either directly or indirectly, any injury, to the pleura, lung, or abdominal viscera. No case of massive collapse involving the entire lung on the side opposite to that wounded has fallen under my observation when a haemothorax was present on the injured side. Whether such cases occur or not, I do not know, but it is possible they occur and that life is not maintained sufficiently long for such patients to reach a hospital on the lines of communication. The contralateral variety of massive collapse involving the whole of one lung is a very remarkable condition, more especially as in many cases the wound on the opposite side of the chest is not only non-penetrating but may be trivial in character, causing no fracture or indeed any extensive injury of the chest-wall. Further, the clinical picture is a simple one, since there are no visceral lesions on the injured side to complicate the physical signs. In such a case the displacement of the heart may be extreme, yet no lesion exists on the injured side capable of displacing it, and thus it is easy to recognize that this displacement is not due to any pushing of the heart over from the accumulation of fluid or gas, but that it is due to the drawing over of the heart owing to collapse of the lung on the side opposite to the injury.

In the homolateral variety the clinical picture is not so simple, and such cases may readily be misinterpreted as cases of haemothorax with rapid absorption of the fluid, as this is often held to account for the fact that the physical signs alter rapidly, and may disappear in a few days.

Massive collapse of the lobar type, partial or complete, is a more common accompaniment of chest wounds, and it may be either unilateral or bilateral in its distribution. The bilateral variety may exist in cases of unilateral wounds of the chest. The contralateral form is a not infrequent complication of haemothorax, but no case has as yet been seen in association with pneumothorax, a fact perhaps not without significance with reference to any theory of the mode of production of collapse.

In lobar collapse the lower lobe of the lung is the part most often affected, 
but cases have been seen where the collapse was limited to the upper lobe, and contralateral collapse may affect the upper lobe alone.

When the lower lobe is involved the physical signs do not suggest that the lesion necessarily begins or is most marked at the extreme base, but rather in the middle third, or even near the apex of the lower lobe. Owing to the upward displacement of the diaphragm and the altered anatomical relations, it is difficult to speak with exactitude as to this point. In the cases where the collapse is partial and involves only a portion of the lower lobe, the upper and middle thirds of the lower lobe are most frequently affected. In some of these cases the collapse is progressive, and ultimately involves the entire lower lobe. Bilateral collapse is, on the whole, uncommon, and usually involves relatively small areas in the lower lobes on both sides, or possibly the entire lower lobe on one side and a portion of its fellow on the opposite side.

\section{The Physical Signs of Massive Collapse.}

The difficulty in the interpretation of the physical signs present depends in part on whether the massive collapse occurs in association with haemothorax, or whether it is the sole lesion produced by the chest wound. It will, therefore, be convenient to consider these signs first in eases where there is no haomothorax present.

(a) Haemothorax absent. The physical signs are usually well marked and easily recognizable, but sometimes, as will be discussed more fully below, the diagnosis may be difficult. The signs are most marked and most easy of recognition in cases of collapse involving the whole of one lung and of the contralateral variety. In such a case the patient presents a non-penetrating wound of the chest-wall limited absolutely to one side, so that there is no question of a foreign body having penetrated and lodged on the side opposite to the point of entry. In some instances the foreign body has been found in the wound, or removed by a simple incision, and direct evidence obtained that the wound is one strictly limited to the chest-wall. Physical examination shows that there is no lesion of the pleura or lung on the wounded side, and this is confirmed by X-ray observation, but very marked and obvious physical signs are found on the opposite side of the chest. Thus, the cardiac impulse, instead of being in its normal position, is displaced towards the affected side or even felt in the axilla, and is also displaced upwards. It may be visible and palpable as high as the third space, and a lateral displacement of two or three inches is by no means rare. The cardiac displacement is most easily observed in the cases where the collapse involves the left lung, and then may be in the axilla, but in right-sided cases the impulse may be detected in the neighbourhood of the right nipple line. The area of visibility of the cardiac impulse may be considerably increased in extent. The affected side of the chest is retracted and immobile, and the dome of the diaphragm on this side is abnormally high and also immobile. The high level 
of the diaphragm is readily detected in the left-sided cases by percussion. On the right side percussion is not so reliable, but $\mathrm{X}$-ray observation yields unequivocal evidence both as to its position and its immobility. Further, physical examination shows that the cases may be divided into two groups. In both, dullness on percussion is present that may extend as high as the clavicle, but in one group the tactile vocal fremitus is diminished or absent, and in the other it is increased. If diminished or absent, the breath-sounds are also diminished or absent, but if the vocal fremitus is increased, then the breath-sounds are loudly tubular or amphoric in character, and bronchophony and pectoriloquy are also extremely well marked. In the majority of cases, even when the cardiac displacement is extreme, the signs conform to the type with increased vocal fremitus and tubular breathing. In otber words, the physical signs in the lungs are those commonly attributed to pneumonic consolidation, but, if anything, the signs are even more marked, especially the tubular and amphoric character of the breathsounds. No real distinction can be drawn between the character of the tubular breathing heard in cases of massive collapse and that present in cases of pneumonic consolidation, but it is possible that the amphoric or hollow character of the sound is more often heard in massive collapse than in pneumonia. Some observers have expressed the opinion that the tubular breathing of consolidation differs from that heard over compressed or collapsed lung, in that in the latter conditions the tubular quality of the sound is heard mainly during expiration, whereas in consolidation it is equally inspiratory and expiratory. This distinction cannot, in my opinion, be maintained, at any rate in so far as concerns massive collapse. One of the main reasons why massive collapse of the lung is so frequently overlooked, is that the tubular breathing is so extraordinarily well developed that its mere presence is at once regarded as conclusive evidence of the existence of pneumonic consolidation. Due regard is not paid to the other signs present, and, more especially, the significance of the cardiac displacement is not recognized.

In some cases, however, dullness on percussion, diminished or absent vocal fremitus, and weakened or absent breath-sounds are the only lung signs present. These cases may also be difficult to interpret when first seen, unless adequate stress is laid on the position of the cardiac impulse. Speaking broadly, these signs are more usually present in the cases seen early, and the later cases tend to show tubular breathing, with increased vocal fremitus. One and the same case, however, may have the one set of signs at one period and the other set at another time, without any material change in the position of the cardiac impulse. Thus, one examination may reveal only weak or absent breath-sounds, and twenty-four hours later loud tubular breathings may be beard over the same area. The cases where tubular breathing is the leading sign are much the easiest to recognize; but it is quite possible that the other group, with the less obvious signs of mere weakness of breath-sounds, is really the more frequent. More observations are required in reference to this point. The signs may not only alter in one and the same case, as just mentioned; but this alteration may occur more than once, the 
weak breathing being replaced by tubular breathing, which disappears to be followed by weak breathing. In such instances there is some resemblance to the well-known signs so characteristic of bronchiectasis, and it is probable that the variations in the signs are dependent on changes in the patency of the bronchial tubes. These recurrent variations are only seen in the later stages of the condition when the chest-wall is no longer immobile, and when there is some degree of re-expansion of the collapsed lung.

Râles and adventitious sounds may be present, but they are often absent throughout the entire duration of even the most extreme cases involving the whole of one lung. On the other hand, they are sometimes abundant, especially in the later stages, when the lung is re-expanding. They are also well marked in the rarer instances, when inflammatory complications develop in the collapsed lung; in fact, the presence of adventitious signs is rather to be associated with re-expansion of the lung or the development of inflammatory complications-they are not essential signs of massive collapse as such.

Displacement of the cardiac impulse is perhaps the most characteristic physical sign of massive collapse of the lung; in fact, this condition cannot be diagnosed with certainty unless this sign is present. The displacement is towards the collapsed lung, and is usually very considerable in amount. In collapse of the left lung, it is not unusual for the cardiac impulse to be felt at its maximum in the mid-axillary line, and in collapse affecting the right lung the impulse can be readily felt in the right nipple line. The patient is not conscious of the cardiac displacement, and does not present any cardiac symptoms. Although the displacement is so great in amount, it is sometimes, even when very marked, of transitory or of short duration, and hence considerable changes in the position of the impulse may occur in periods as short as twenty-four or forty-eight hours. This is not always the case, and the return of the heart to its normal position may be very slow and take as long as three or even four weeks; more usually, however, the return to normal takes place in about ten days. In the cases of extreme displacement of the heart, the signs in the lungs are usually not only well marked, but are of the type characterized by the presence of tubular breathing, increased vocal fremitus, together with bronchophony and pectoriloquy. This, however, is not invariable, and extreme displacement may occur, accompanied only with weak or absent breath-sounds at the base towards which the heart is displaced. This is important, since were it not for the cardiac displacement the physical signs present might well escape full recognition. Thus, in one case of a contour wound of the left chest, where the patient presented no urgent symptoms of any kind, the apex beat was found to be in the right nipple line, but the signs at the right base were little more than weakened breathsounds. These signs were so ill marked at the first examination as to suggest the possibility of the case being one of dextrocardia. Twenty-four hours later, the signs at the right base were well marked, the tubular breathing being very distinct, and the case was clearly one of massive collapse. This was confirmed by X-ray examination and by the subsequent course of the case, since the heart 
returned to its normal position, and at no time was there any lesion of the left pleura or lung.

Although displacement of the heart is such a marked sign of massive collapse, and is often so great in amount, yet, so far as my experience goes, it is not accompanied with the presence of basal cardiac murmurs, although such murmurs have been thought by many to result from mere displacement.

The displacement of the heart is mainly lateral, but in cases where the collapse involves either the whole lung or the upper lobe, there is also displacement upwards, so that the maximum impulse may be felt in the third space or behind the third rib. This upward displacement is more easily observed in cases of collapse involving the left lung.

The heart returns towards its normal position before the signs in the lung have disappeared, and it is by no means uncommon for the impulse to reach its normal site, whilst there is still an area of variable size in the scapular region where dullness and tubular breathing are still present, and these lung signs may persist for three or four weeks in some cases.

The persistence of such signs as tubular breathing, bronchophony, and pectoriloquy, at a time when the heart has returned towards its normal position, or even has reached it, is further evidence that the most important auscultatory sign of massive collapse is either weakness or absence of breath-sounds, since signs such as tubular breathing and bronchophony can evidently be extremely well marked when the degree of collapse is no longer great nor the area involved large. In other words, a small area of collapse, not sufficient to cause marked displacement of the heart, may, provided bronchial tubes of a suitable calibre are patent, give rise to extremely well-marked physical signs.

Weakness of the breath-sounds is a sign that may be due to many causes, and thus it often does not attract the attention it deserves; but in cases of massive collapse it derives its importance from the signs associated with it, especially the retraction and immobility of the chest-wall, together with the displacement of the heart and diaphragm. Considered by itself, it would often be difficult to appraise its true significance, but its associated signs reveal at once that it is really due to complete cessation, or great diminution, of the movement of air in and out of the affected lung.

Although the physical signs are most marked and most easily interpreted in cases of massive collapse involving the entire lung, yet they are also comparatively easy to recognize in cases of collapse involving an entire lobe (usually the lower lobe) on the side opposite to that wounded, and where the wound is single, unilateral, and limited to the parietes. In such cases the signs are essentially similar to those just described, except that they are more limited in area and correspond to the lower or upper lobe as the case may be. The cardiac displacement, although always well marked, is not so great as in cases of collapse involving the entire lung.

(b) Haemothorax present. When massive collapse occurs in association 
with haemothorax in cases of unilateral wounds of the chest, it is much more difficult to recognize, and this applies both to the homolateral and the contralateral varieties.

Homolateral massive collapse. In this variety, although only a small basal haemothorax is present, the physical signs involve the whole of the wounded side. Dullness, weak or tubular breath-sounds, may extend as high as the clavicle, and unless care is taken to avoid the error, it may be thought at first that there is a very large collection of fluid in the pleural cavity. Examination may reveal, however, that the cardiac impulse is not displaced outwards, but that it is either normal in position, or, in many cases, actually displaced towards the affected sile, instead of away from it. The position of the cardiac impulse is not consonant with the extensive physical signs being really due to a large amount of fluid in the pleura. Further examination shows the chest on the affected side to be retracted to a greater or less degree, and immobile, and the diaphragm is also found to be high and also immobile on that side. Another very important point is the fact that the physical signs alter rapidly, and in the course of a few days, sometimes even of a period as short as twenty-four or forty-eight hours, the lung becomes again expanded, and the only signs present are those indicative of a quite small effusion at the base. Such cases are not infrequently interpreted erroneously as instances of the rapid absorption of the bloody exudate from the pleura. There is no evidence that the pleura can absorb blood in the short pcriods that elapse in these cases, and conclusive evidence that the condition present is really collapse and not a large haemothorax is afforded by $\mathrm{X}$-ray examination, quite apart from the fact that the peculiar and transitory physical signs present are fully and adequately explained by the presence of collapse. Further, in many cases, exploratory puncture has shown no fluid over the region where the signs were marked.

Contralateral massive collapse. In cases of unilateral haemotborax, no case of massive collapse involving the entire lung on the opposite side has been seen, and it is probable that such cases, if they occur, would not survive sufficiently long to reach a base or line of communication hospital. On the other hand, cases of contralateral lobar collapse are by no means uncommon, although the diagnosis of such cases is often difficult. The reason of this difficulty is obvious, since the displacement of the cardiac impulse is the physical sign of most significance in the diagnosis of massive collapse, and when haemothorax is present any cardiac displacement will naturally be attributed to the haemothorax, and thus the physical signs in the lung are regarded as due to the existence of a contralateral pneumonia when their true cause is a contralateral lobar collapse. In most cases the absence of the symptoms of pneumonia, together with a rapid change in the position of the heart, without any treatment such as paracentesis of the haemothorax, reveal the true nature of the case. Further, retraction and immobility of the lower chest, together with the upward displacement of the diaphragm, are signs that assist very materially in the diagnosis. 'l'he existence of contralateral collapse and its subsidence are very important factors in causing 
rapid alterations in the position of the heart in cases of haemothorax where no paracentesis has been performed.

In some cases of contralateral collapse associated with haemothorax the physical signs are only found over a limited area, between the vertebral border of the scapula and the spine, corresponding roughly with the middle third of the lower lobe of the lung. This area is the same as that in which the signs remain most persistently in cases where originally the collapse involved either the whole of one lobe, or even the entire lung. It would seem, therefore, that this is an area in which the signs are not only most readily recognized, but also where they are most lasting.

In this description of the physical signs associated with massive collapse we have, so far, more especially considered the following types: Contralateral collapse, either total or lobar, the former as yet only seen with unilateral parietal or contour wounds, the latter occurring as an association not only of unilateral contour wounds, but also of unilateral penetrating wounds, causing a haemothorax on the wounded side.

Homolateral massive collapse, involving the entire lung, and associated with a small and negligible haemothorax, has also been described. But there is yet another form of massive collapse that merits attention, as it produces a remarkable clinical picture. In this last variety, as a result of a unilateral penetrating wound, a haemothorax of moderate or even of a large size is present, yet the following are the physical signs observed: The chest on the injured side is markedly retracted and immobile, there is dullness on percussion, with increased vocal fremitus, together with extremely loud tubular or amphoric breathing, and bronchophony and pectoriloquy are well marked. The diaphragm is raised on the affected side, but the heart's impulse is displaced away from the injured side; it may be as much as two inches from its normal position. The striking feature of these cases is the fact that, notwithstanding the retraction of the chest-wall on the injured side, the heart is displaced in a direction similar to that seen in pleural eftusion, i.e. away from the affected side. This is the only variety of massive collapse in which the heart is not displaced towards the affected lung, and the reason is, of course, that the collapse is accompanied by the presence of a considerable amount of fluid. The importance of this type of case lies in the fact that the presence of even considerable amounts of haemothorax fluid, instead of leading to bulging of the chest with cardiac displacement towards the opposite side, causes, it is true, the cardiac displacement, but this is associated with marked retraction of the chest-wall over the pleural fluid. In some cases this chest retraction is only of temporary duration, and with a further increase in the pleural exudate, owing to the presence of infection, the side becomes bulged, and the case then presents the ordinary signs of a large pleural exudate. Such eases would seem to support the view that the collapse present in cases of haemothorax cannot be regarded as merely dependent upon the presence of the fluid in the pleura, since, if this were so, it is not clear why retraction of the chest-wall should be such a marked feature. 
The study of cases of pure massive collapse, without haemothorax, whether contralateral or homolateral in type, serves to interpret the phenomena seen in cases of simple haemothorax, although in these cases of haemothorax the clinical picture is necessarily more complex. The actual signs present in any case must depend, to some extent, on the relative proportion of fluid on the one hand, and massive collapse on the other, and these factors are of special importance in determining the position of the heart.

\section{Symptoms.}

The symptoms vary considerably in severity in different cases, and certainly do not seem to be dependent upon the extent of the massive collapse presont, since cases where the whole of one lung is involved may be seen where the patient has few, if any, symptoms, provided he is in bed and at rest.

In most cases there is some dyspnoea, usually moderate in amount, but markedly increased on slight exertion, such as sitting up in bed, or turning over on one side, and the respirations may bo somewhat increased in rate. No undoubted instance of the severe and urgent dyspnoea simulating that seen in pulmonary embolism has been seen; but in one case, where urgent symptoms of this type developed, they may have been due to massive collapse. In this instance, in a case with a large sterile haemothorax, very urgent dyspnoea supervened with great suddenness, together with signs of apparent consolidation on the side of the haemothorax. They ultimately subsided with no special treatment, and it was very improbable that they were due to pneumonia, and they were certainly not due to infection, as the patient recovered after paracentesis. The case, however, cannot be regarded as one in which the existence of massive collapse was proved. It is remarkable that urgent symptoms have always been absent in the cases of collapse involving the whole of one lung of the contralateral type, associated with contour wounds. In many of these cases the patients have only suffered from slight cough and dyspnoea. In some there has been some expectoration, but in several of the most marked examples of the condition there has been no expectoration at all. On the other hand, where the collapse is less extensive, e. g. contralateral lobar collapse, a more or less copious muco-purulent expectoration sometimes develops, and may persist for several days. The expectoration may somewhat resemble that of purulent bronchitis, but it is usually more watery and by no means so copious. A rusty sputum has never been present in any case diagnosed as massive collapse, except when pneumonia affecting the collapsed lung has occurred as a complication, and this sequel has been observed in several instances, and on two occasions has been confirmed on post-mortem examination. It is important to recognize that massive collapse may run its whole course without the presence of any expectoration, and with little or no cough.

The duration of massive collapse is variable; in some cases the signs not only alter, but may even clear up within a few days. Thus the heart may return 
towards its normal position after a displacement of the impulse of as much as two inches in from one to three days, but it is of some interest that, even in these cases where the return of the heart to its normal position is not only rapid but also complete, some signs of the pulmonary collapse may persist, such as retraction, limitation of respiratory movement, and a localized area of tubular breathing. The more usual course of these cases is much slower, and the return to the normal takes place gradually, and is not usually complete until the lapse of three weeks from the onset; in some cases it may even be longer. The immobility of the side disappears, and limited or imperfect expansion takes its place; the apex beat returns gradually, and over the area of lung collapse the breath-sounds become less tubular. As the re-expansion of the lung takes place, adventitious sounds may become very abundant, especially crepitations, fine or medium in character, and râles, and these signs often present a considerable resemblance to those heard in a resolving pneumonia. During this stage of re-expansion, cough and expectoration are often present, but not invariably the expectoration is usually watery and mucoid, sometimes purulent, but never rusty. In exceptional cases it may not only be watery but also abundant, and rather suggestive of oedema of the lung, but true oedema of the lung has not as yet been observed post mortem in such cases; on the other hand, such lungs show evidence of marked congestion.

\section{Complications.}

Certain complications may supervene, and involve the portion of the lung affected with massive collapse; bronchitis, pleurisy, and pneumonia are definite and certain inflammatory complications that may occur, and of which clear clinical and post-mortem evidence bas been obtained. It is probable that in some cases oedema of the affected lung develops as a complication, but the evidence of this is not as yet complete, although, as mentioned above, certain physical signs seen during the stage of re-expansion of the lung, and associated with a copious mucoid or watery sputum, are very suggestive of the presence of oedema.

Purulent bronchitis undoubtedly occurs as a complication, as it may be limited to the collapsed lung. In fact, this is the main reason for regarding it as a complication and not as a causative factor in the production of the collapse. The presence of purulent bronchitis limited to, or better marked in, the collapsed lung, as seen post mortem in cases of massive collapse, is a fact in striking contrast to the now well-known fact that where purulent bronchitis develops as a complication of haemothorax there is usually far less bronchitis in the lung beneath the haemothorax than in its fellow. The lung collapsed in association with haemothorax is not prone to develop inflammatory complications, but the lung of massive collapse may and frequently does develop such infections.

Pleurisy is not uncommon as a late complication of massive collapse. It is usually of the dry variety, giving rise to a friction rub; but effusion may occur, and in one case this was sufficient in amount to render paracentesis advisable. 
Usually the effusion is very moderate in amount, and the correct interpretation of the case would be difficult, unless it had been under observation prior to the onset of the effusion.

Pneumonia. Two cases of lobar pneumonia supervening as a complication of massive collapse have been observed, and in both cases the pneumonia was limited to the collapsed lobe, and the patients had been under observation for many days prior to the onset of the pneumonia with typical physical signs of massive collapse of the lower lobe. When the pneumonia developed the heart's apex beat returned towards its normal position. One case was fatal, and at the post-mortem examination lobar pneumonia, in the stage of uniform grey hepatization, was found, but the lobe involved was still, notwithstanding the pneumonic consolidation, distinetly smaller than its normal size.

\section{Diagnosis.}

The difficulty in the diagnosis of massive collapse varies greatly in different eases ; the most sharply defined clinical picture is that seen in a unilateral nonpenetrating wound of the chest, where massive collapse of the entire lung is present on the side opposite to that wounded, i. e. contralateral massive collapse. On the other hand, a small area of contralateral collapse occurring as a complication of a case of infected haemothorax may be difficult to distinguish from a contralateral pneumonia. This arises from the fact that pyrexia and bloody sputum may both result from the injury to the chest and lung on the wounded side, and not from the supposed pneumonia. Further, the cardiac displacement is apt to be attributed solely to the haemothorax pushing the heart over, when in reality it is mainly dependent on the contralateral massive collapse. Notwithstanding the difficulties, the condition can usually be recognized with accuracy, provided its possible occurrence is borne in mind and care is taken during the examination of the chest. The position of the cardiac impulse, the size of the chest on the affected side, the immobility of the chest-wall locally or generally, together with the high level of the dome of the diaphragm on the affected side, are signs of the utmost significance and value. The following errors of diagnosis have been made in cases where the real condition present was contralateral massive collapse, involving either the whole lung or one lobe. In one case a subphrenic collection of gas and fluid was supposed to be present in order to account for the upward displacement of the heart and diaphragm on the side of the collapsed lung, whereas not only was there no abdominal lesion, but the wound was a trivial non-penetrating one of the opposite side of the chest, and the case made a complete and uneventful recovery. In another case a pneumothorax was supposed to be present on the wounded side in order to account for the great cardiac displacement, although the patient presented neither signs nor symptoms of pneumothorax, and the wound was one that did not implicate the pleura. In another case the collapsed lung was actually explored by excising a rib, as owing to the well-marked physical signs in the lungs, tubular breathing 
and râles, it was supposed that the lung was breaking down and that a grave pulmonary lesion was present. In another case the displacement of the cardiac impulse was attributed to cardiac dilatation and the signs of pulmonary collapse entirely overlooked. Most of these errors would be avoided if proper attention were paid to the question of displacement of the cardiac impulse, and especially if all observers recognized that displacement of the cardiac impulse is not necessarily dependent upon the heart being pushed over, but that it may be due to its being drawn over. Or to put the matter more correctly, the morbid process leading to the cardiac displacement should be sought for, and may possibly be found on the side towards which the heart is displaced, rather than on the opposite and wounded side. Further, it must not be too readily assumed, even when a displacing agent such as a haemothorax is present on the wounded side, that this is the sole cause of the cardiac displacement present. If no signs indicative of a pleural lesion are found on the wounded side, careful examination of the opposite side should be made, if the cardiac impulse is displaced.

In most cases of massive collapse, the heart is not only displaced, but it is greatly displaced, and this fact alone should cause a most careful examination to be made on both sides of the chest, notwithstanding the existence of a simple unilateral lesion and even in the absence of urgent symptoms. One difficulty sometimes encountered is the fact that the pulmonary physical signs may be comparatively slight, even when the cardiac impulse is greatly displaced, and then sufficient importance may not be attached to them; thus, in collapse of the right lung, the impulse may be in the right nipple line, but the only signs at the right base in an early period of the lesion may be weakness or absence of breathsounds. Such cases may at first be misinterpreted as instances of dextrocardia, but the lapse of a few days, or sometimes of hours only, reveals their true nature, as the signs nlter, and ultimately the heart returns to its normal position. The upward displacement of the diaphragm is a sign of the greatest importance, and is easily detected on the left side by the greatly increased stomach resonance. This increased resonance may lead to error, in that it may be attributed to pneumothorax or to a subphrenic collection of gas. Such mistakes are readily avoided by paying attention to the direction of the cardiac displacement, and also from the fact that the affected side is retracted instead of being bulged.

When the massive collapse involves the entire lung, the displacement of the cardiac inpulse is oblique, inasmuch as it is displaced vertically as well as laterally. This very characteristic sign is more obvious in cases of collapse involving the left lung. In collapse limited to the upper lobe of the lung, the cardiac displacement is also oblique.

In cases of homolateral massive collapse, especially if associated with a small haemothorax on the injured side, the diagnosis is often difficult, and two errors are more especially liable to be made. In the first place, a large haemothorax may be diagnosed owing to the extent of the physical signs, whereas in reality there is only a very small collection of fluid. The second type of error is more serious, and is especially apt to be made in cases where infection of the haemothorax is 
present, and the patient consequently presents more or less urgent symptoms. In such a case the well-marked signs may lead to a diagnosis of pneumonic consolidation, and the symptoms due really to the infection of the pleural contents may be attributed to the supposed presence of pneumonia. If this error is made, valuable time may be lost, and the patient's chance of recovery gravely imperilled. The usually accepted signs of consolidation, i. e. dullness on percussion, increased vocal fremitus, and tubular breathing, are often extremely well marked in cases of massive collapse, and if, in addition to these, pyrexia, hurried respirations, and a bloody sputurn are present, it is not surprising that a diagnosis of 'traumatic pneumonia' is made, whereas the true condition present is really a small infected haemothorax together with a large area of massive collapse. Such cases require immediate surgical intervention, and thus call for early exploration in order to determine whether fluid and infection are present in the pleural cavity. The retraction and immobility of the affected side are signs of value in the diagnosis of this type of case, but the position of the cardiac impulse varies, and is dependent upon the size of the haemothorax in association with the massive collapse. Thus, if the effusion is of some size, the heart may be displaced, as in ordinary pleural effusion, towards the sound side, but even then the presence of massive collapse may be detected owing to the retraction of the affected side. If the haemothorax is small, the heart may be displaced towards the affected side, and then the diagnosis of massive collapse is easy. In pneumonic consolidation the side affected is not retracted, and the cardiac impulse is not materially displaced, and certainly is not displaced towards the side affected.

The differential diagnosis between a large haemothorax and a small haemothorax associated with massive homolateral collapse involving the entire lung can also generally be made, owing to the presence of retraction of the chest-wall in the one case, and bulging in the other, and also because the heart is not only not displaced away from the affected side, but on the contrary may be displaced towards it. Further, in homolateral massive collapse the dullness extends right up to the clavicle, whereas even in the very largest haemothorax there is, at any rate in most cases if not in all, a small area of skodaic hyper-resonance in the uppermost intercostal space or spaces. Although the diagnosis of massive collapse can usually be made from a consideration of the physical signs alone, yet there are other factors that may be of help. Thus, in many cases of massive collapse involving the entire lung, the patient may present no symptoms beyond slight dyspnoea; in other words, although the physical signs are most extensive, symptoms are absent or slight in amount. This is in great contrast to what obtains when widespread pneumonia, or a very large haemothorax is present. It is true that in the earlier stages of massive collapse dyspnoea may be present, and even be urgent, and in the later stages, when re-expansion is taking place, a copious mucoid sputum is sometimes seen; but these signs are not invariable, and in the fully established condition all marked symptoms may be absent.

Pyrexia is a sign of very uncertain value; there is some evidence that it may 
be associated with massive collapse, and it is certain that in a few cases pleurisy and pneumonia may occur as complications. Further, in any given case the pyrexia may be due to other causes, $\theta$. g. the state of the wound, the presence of an infected haemothorax, \&c.; hence the presence of pyrexia cannot be regarded necessarily as evidence that the signs present are due to pneumonia rather than to massive collapse. The absence of pyrexia, however, when the signs are marked and widespread, is a factor of value and importance in the diagnosis.

For diagnostic purposes the physical signs may be divided roughly into three groups, corresponding to three stages in the progress of the case. In the first period the main physical signs are retraction and immobility of the affected side, together with weakness or absence of breath-sounds and displacement of the heart, often extreme in amount. In the second period the weakness of the breath-sounds has been replaced by loud tubular or amphoric breathing, together with increased vocal fremitus and loud bronchophony and pectoriloquy. In the third period, i.e. the stage when the lung is expanding, abundant râles and crepitations may be present over the area where tubular breathing is marked. In both the second and third stage the heart is still displaced, but, as already mentioned, the lung signs may sometimes persist over a small area at a time when the heart has returned to its normal position.

The recognition of contralateral massive collapse is most easy in cases of unilateral non-penetrating wounds of the chest and in cases of penetrating wounds, where the entry and exit wounds are in such positions as to prove that the injuries are confined to one side of the chest. In cases where there is only a wound of entry, and the position of the retained missile is doubtful, the difficulties of diagnosis are far greater. The presence of bilateral physical signs may be dependent upon the existence of direct lesions in both pleural sacs and their contents, as well as upon the presence of a unilateral injury and the subsequent development of a complication like massive collapse. In many of these cases it is not possible to diagnose with certainty between effusion and collapse without exploratory puncture of both pleural cavities. The position of the cardiac impulse is again the most valuable sign, since in bilateral haemothorax great displacement is not usual; hence if the signs suggest a moderate haemothorax on one side, and yet there is great displacement of the apex beat, it is probable that contralateral collapse rather than bilateral haemothorax is present.

$\mathrm{X}$-ray examination is necessarily of great value, and should be used in all cases.

\section{Aetiology.}

Although this communication deals with massive collapse more especially in its relation to gunshot wounds of the chest, in any discussion as to its causation and nature it must be recognized that it also occurs as a complication of a number of other injuries, and, indeed, in certain diseases. In many of these 
conditions, however, the problem is more complicated than in those where it follows an injury of the chest. Thus it is not infrequent in wounds of the abdominal wall, and also in wounds of the buttocks, pelvis, and thighs. Although undoubted instances have been met with in cases of gunshot wounds of the thigh, with fracture of the femur, it would seem to be a rare complication of this injury. In cases following wounds of the buttocks or pelvis, the wound is often of a severe character, and such cases are not very suitable for the study of the mechanism of its production, in that the wound is often of such a character as to bave led to the patient being compelled to assume a constrained and unnatural posture for hours or even for days, and it is extremely difficult to determine how important a factor this may be in the production of the lung complication. It will suffice to say here that massive collapse, seen after these injuries, is essentially similar in character to that seen after injuries of the chest, and, therefore, that the condition is not to be regarded as solely produced by chest injuries. Further, two typical cases were observed in unwounded men where the condition occurred as a sequel to ordinary lobar pneumonia, and where it cleared up completely and rapidly, and was, therefore, not dependent upon any chronic inflammatory thickening of the pleura or any fibrosis of the lung produced by the pneumonia. These cases, however, will not be discussed further, and are only mentioned in illustration of the fact that the condition is not limited to injuries. Many cases of collapse as a result of abdominal operations, appendicitis, hernia, \&c., have also been seen, similar to those described by Pasteur, but in these cases also the problem is complex owing to many factors, such as the influence of an anaesthetic, the presence of peritonitis, \&c. As already mentioned, the writer has had no opportunity of determining the frequency of its occurrence as a complication of abdominal wounds, but it eertainly occurs in wounds of the abdominal wall, buttocks, ilium, and thighs, where there is no lesion of the abdominal or pelvic cavities.

In wounds of the chest the outstanding fact is that massive collapse in its most extreme form, involving the whole of one lung, may occur as a sequence to a small wound limited absolutely to the chest-wall. Therefore injury to the lung is not necessary for its production. Further, it occurs not infrequently on the side of the chest opposite to that injured, even when the wound is of this trivial character, and the collapse involves the entire lung, and this is further evidence, if such is required, that injury of the lung itself is not necessary for its production. It may be completely developed in less than twenty-four hours after the infliction of the wound; how soon it develops after the wound is not known, but no case has been seen by the writer where collapse was known to be absent when the patient was admitted to hospital and subsequently developed. In other words, all cases had signs on admission, but very few cases have been seen earlier than twenty-four hours after being wounded. A few cases have been seen where the signs suggested that the lesion was progressive, but this is exceptional, and it is possible that the observations were not strictly accurate. The condition, even in its most extreme form, may occur without the patient 
giving any history of expectoration either of blood or sputum, purulent or otherwise. Three points seem to be of importance in any discussion as to its causation, i. e. (1) that it may follow trivial injury of the chest-wall, (2) that it always occurs early, and (3) that there may be no expectoration at any stage in its evolution, and sometimes no urgent symptoms. When massive collapse is present the chest on the affected side is immobile, and the problem as to its causation would seem to centre round the significance to be attached to this immobility.

Is the chest immobile and retracted because the lung is collapsed, or is the lung collapsed because of the immobility and retraction of the chest-wall? This problem is difficult of solution from the study of clinical phenomena alone, and probably will require experimental methods before a satisfactory answer can be given; but yet some light may be thrown on the question by a discussion of the clinical facts. If the collapse is primarily of lung origin, the only obvious mode of production would seem to be that of obstruction of the bronchi. There is no evidence, in these cases of massive contralateral collapse involving the entire lung, of any gross obstruction of the larger bronchi. It is difficult to see how a unilateral injury, limited to the chest-wall, in a healthy man, could within twenty-four hours produce an obstruction of the main bronchus of the opposite lung sufficient to cause complete collapse of the lung, and yet that no urgent symptoms and no expectoration should be present. In cases where the wound is a penetrating one and involves the lung, there must always be the possibility that blood, perhaps in quantity, may have been inhaled into the opposite lung, and hence, in discussing the mode of production of massive collapse, only the cases of contralateral collapse with non-penetrating wounds are considered. Further, there is definite post-mortem evidence that massive collapse may be present, and yet no gross obstruction of the main bronchi is found. Hence obstruction of the main bronchi may be excluded as a common or probable cause of the condition.

The problem whether obstruction of the smaller bronchi, or bronchioles, is the essential factor is much more difficult. Such a mechanism would probably be a sufficient explanation of the condition, if we could surmise how it could be calied into action. Massive collapse is not a condition produced by the more common and familiar causes of bronchial obstruction. Thus, in bronchitis in its many forms, where great obstruction to the finer bronchi is present, although collapse in limited small areas is common, yet passive collapse does not, so far as I know, occur; and in a considerable number of fatal cases of purulent bronchitis I have never seen it. Further, it is remarkable that so-called compensatory emphysema is not a marked feature of massive collapse, whereas it is a most characteristic accompaniment of the limited areas of collapse associated with bronchitis.

Again, certain varieties of asthma are supposed to be dependent upon bronchial spasm, but here, also, massive collapse is not a sequel. Finally, all forms of bronchial obstruction hitherto known always produce severe and often dangerous symptoms, and this is certainly not true of all cases of massive collapse, 
even when they involve very large areas of the lung, or even the entire lung. The patient from whom Plates 10-12 were obtained was admitted to hospital on the evening of the day on which he had been wounded, and had massive collapse involving the whole of the left lung; he had no urgent symptoms and had walked four miles after being wounded.

Although it must be admitted that obstruction of the finer bronchi, produced perhaps by an increased tonic contraction of their musculature, might possibly be held capable of producing the condition, such an explanation is purely hypothetical, and there are, as mentioned above, grave clinical difficulties in the way of its acceptance.

There are also serious difficulties if the matter is considered from the standpoint of physiology. Thus any diminution in the volume of the lung, brought about by diminished pressure in, or obstruction of, the bronchial tree, immediately brings into play increased inspiratory action and increased inspiratory tonus. This mechanism is very probably the cause of, or one of the causes of, the production of so-called emphysema in such cases. In collapse retraction and immobility of the chest and of the diaphragm are not only leading phenomena, but are also early phenomena. They are not late and secondary results of the collapse; they are present in their most marked form in the cases when first seen, and they are very difficult of explanation on any hypothesis of the lung collapse being primary and dependent upon bronchial obstruction.

For these reasons it seems probable that bronchial spasm or bronchial obstruction cannot well be regarded as the cause of the variety of massive collapse dealt with in this paper, and more especially of massive contralateral collapse in non-penetrating chest wounds.

If the collapse is not primarily of bronchial origin, we must next consider whether it is probable that it arises as a result of the immobility and retraction of the chest-wall, and discuss further how this chest-wall condition itself arises. It is well known that inefficient expansion of the chest, however produced, is capable of causing collapse in varying degrees of the underlying lung. In some instances a constrained posture or prolonged recumbency is sufficient to cause quite extensive collapse, involving, for instance, one lobe of the lung. Pasteur, in his article on massive collapse as a complication, especially of abdominal operations, suggested that the inspiratory mechanism was in abeyance and that the expiratory muscles acted as actual deflating agents. There are difficulties in the way of the complete acceptance of this view, inasmuch as it is necessary to apply very considerable pressure to the exterior of the lung in order to expel the contained air, but accepting Pasteur's conclusion that the inspiratory mechanism is in abeyance in these cases, there is another way in which it is possible that collapse might be produced. If, as a result of the cessation of inspiratory movements, the chest became fixed in an extreme expiratory phase, the air in the lungs would not be adequately renewed, and the air present would be absorbed by the blood-stream and thus collapse might be brought about. The volume of the lung in massive collapse is always much less than that of the lung 
during the most extreme expiration, and this also is a reason for thinking that something more than expiratory deflation is necessary for its production.

Physical and X-ray examination reveal that the chest-wall and diaphragm are immobile and in an extreme expiratory phase, the immobility of the diaphragm being especially obvious. A consideration of the following facts supports the view that the chest-wall condition is primary and not secondary to the lung condition. In certain cases of haemothorax, where homolateral massive collapse is present, the heart, as already pointed out in the section dealing with physical signs, is displaced towards the opposite side, and yet the affected side is retracted and not bulged. If the chest-wall condition was merely passive and only dependent upon the existence of the collapsed lung, it is very difficult to understand why the pleural effusion does not cause bulging of the side. The coexistence of retraction of one side, with displacement of the heart to the opposite side, certainly suggests that the chest-wall condition is an active and not a passive one.

The mode of production of this chest-wall condition remains most obscure. In ordinary wounds of the chest its presence might be regarded as somewhat similar to the state of the abdominal wall in abdominal injuries and diseases. It might even be regarded as of teleological significance and eminently a safeguard to the wounded lung. The phenomena of contralateral collapse, as a complication of trivial parietal wounds, is a very serious objection to any such explanation, as is also the occurrence of similar collapse as a result of wounds in distant parts of the body. The immobility and retraction of the chest-wall, even in the homolateral cases, are not dependent upon the presence of pain, \&c., produced by the severity of the wounds, as the wound is often of a quite simple character. In the contralateral cases it is obvious that some more complex mechanism is at work, since no injury is present on the affected side. No case of contralateral collapse has fallen under my observation as a complication of pleural effusion, nor yet in pneumothorax, even when the latter has been the result of a gunshot wound. It is possible that it may occur in pneumothorax, but that such cases are rapidly fatal, or it may be that the urgent dyspnoea present in the early stages of pneumothorax prevents its development.

It would seem in the present state of our knowledge that massive collapse is a condition that may supervene at an early stage, after an injury of the chest or other part, and that the causative injury is not necessarily a severe one. Further, massive collapse in its most marked form, i. e. contralateral massive collapse involving the entire lung, may develop when the injury is not only trivial, but is also limited absolutely to the parietes. The collapse is more readily explained as a result of the immobility and retraction of the chest-wall and diaphragm, than if regarded as due to bronchial obstruction. The mechanism by which this condition of the chest-wall is produced is obscure, but possibly it is of reflex nervous origin. 


\section{Contralateral Massive Collapse involving entire Lung from Non-penetrating Parietal Wound.}

Private R. G- . Aged 41.

16.5.17. Wounded on the morning of 16.5.17. No haemoptysis. Walked four miles to dressing station, where a piece of shrapnel fell out of the wound on taking off his tunic. Admitted to General Hospital same evening. Several slight superficial wounds of skin and face and right back, one only involving deeper parts, $1^{\prime \prime}$ external to angle of right scapula, about $1 \frac{1}{2}$ " long, from which foreign body had fallen. No symptoms, and patient says he is quite well. Left side of chest immobile and heart displaced to left.

18.5.17. Left chest immobile. Heart's apex beat on level of nipple and $1^{\prime \prime}$ external to it, and $5^{\prime \prime}$ to left of mid-sternum. Whole of left chest dull on percussion as high as clavicle.

Resonance in 'Traube's space well marked. Tubular breathing, bronchophony, and pectoriloquy.

X-ray report: Considerable opacity over whole of left chest. No signs of fluid on right side. Right side diaphragm moving well, left side diaphragm immobile.

20.5.17. Chest explored by puncture both sides. On right side small quantity of bright blood, probably lung blood. On left side puncture at three separate spots; negative result.

21.5.17. Circumference of chest, $34 \frac{1}{2}{ }^{\prime \prime}$ to $36^{\prime \prime}$. Right side, $17 \frac{1}{2}{ }^{\prime \prime}$ to $18 \frac{1}{4}$ ". Left side, $17^{\prime \prime}$ to $17 \frac{3}{8}^{\prime \prime}$.

Slight cough. Left chest moving slightly. Apex beat $5^{\prime \prime}$ to left of midsternum, and slightly above level of nipple. Dullness to second rib on left side. Breath-sounds feeble over left chest. a little.

22.5.17. X-ray report: Left apex clearer and diaphragm on left side moving

23.5.17. Abundant râles in vicinity of angle of left scapula. Temperature normal. Pulse 72.

24.5.17. X-ray report: Right diaphragm moving well, left only moving a little; level of diaphragm on this side at fifth costal cartilage.

26.5.17. X-ray report : Diaphragm moving well on both sides. Left lung clear almost to base. Heart still displaced. Left diaphragm at the level of fifth costal cartilage.

28.5.17. Impulse in fourth space $\frac{3^{\prime \prime}}{4}$ external to nipple. Chest-wall still retracted and $X$-ray report gives level of left diaphragm at fifth intercostal space.

2.6.17. Some impaired resonance still present at angle of left scapula, with a small area of tubular breathing, increased vocal fremitus, and bronchophony. Normal anteriorly. No cough and no expectoration. Impulse in nipple line.

6.6.17. Evacuated to England quite well.
Three X-ray Plates.
(1) 19.5.17.
(2) $21.5 .1 \%$
(3) 23.5.17.

\section{Massive Collapse of Left Lung following a Wound of Left Ilium.}

Private Basil H.S_-. Aged 22 years.

Wounded 26.9.16, and was lying out three days.

Dyspnoea immediately after being wounded.

Admitted to General Hospital on 2.10.16.

Entrance wound healed $2^{\prime \prime}$ inside anterior superior iliac spine on the left side. Exit 1" below crest of ilium and a hand's breadth from middle line. 
Temperature normal. No abdominal signs or symptoms.

Respirations 30. Pulse 60.

Apex beat external to left nipple line.

Left chest retracted and movement very slight.

Resonance impaired, breath-sounds feeble.

Behind tubular breathing up to angle of scapula with bronchophony. No adventitious sounds. Slight cough and some mucoid watery expectoration.

6.10.16. X-ray report: Opacity over left chest, especially at the base. Heart displaced to left, and on left side diaphragm raised and immobile.

15.10.16. X-ray report: Translucency much increased on left side, but heart still displaced. Diaphragm moving on both sides.

This patient received no special treatment and the signs were so definite that the chest was not punctured. He was evacuated to England on 16.10.16, much improved and presenting no symptoms.

\section{DESCRIPTION OF FIGURES.}

Prates 10,11, 12. Three X-ray photographs taken in the sitting posture from the case of Private R. G- quoted above.

Plate 10 taken on 19.5.17. Plate 11 taken on 21.5.17. Plate 12 taken on 23.5.17. 
Vol. 12 Pl. 10

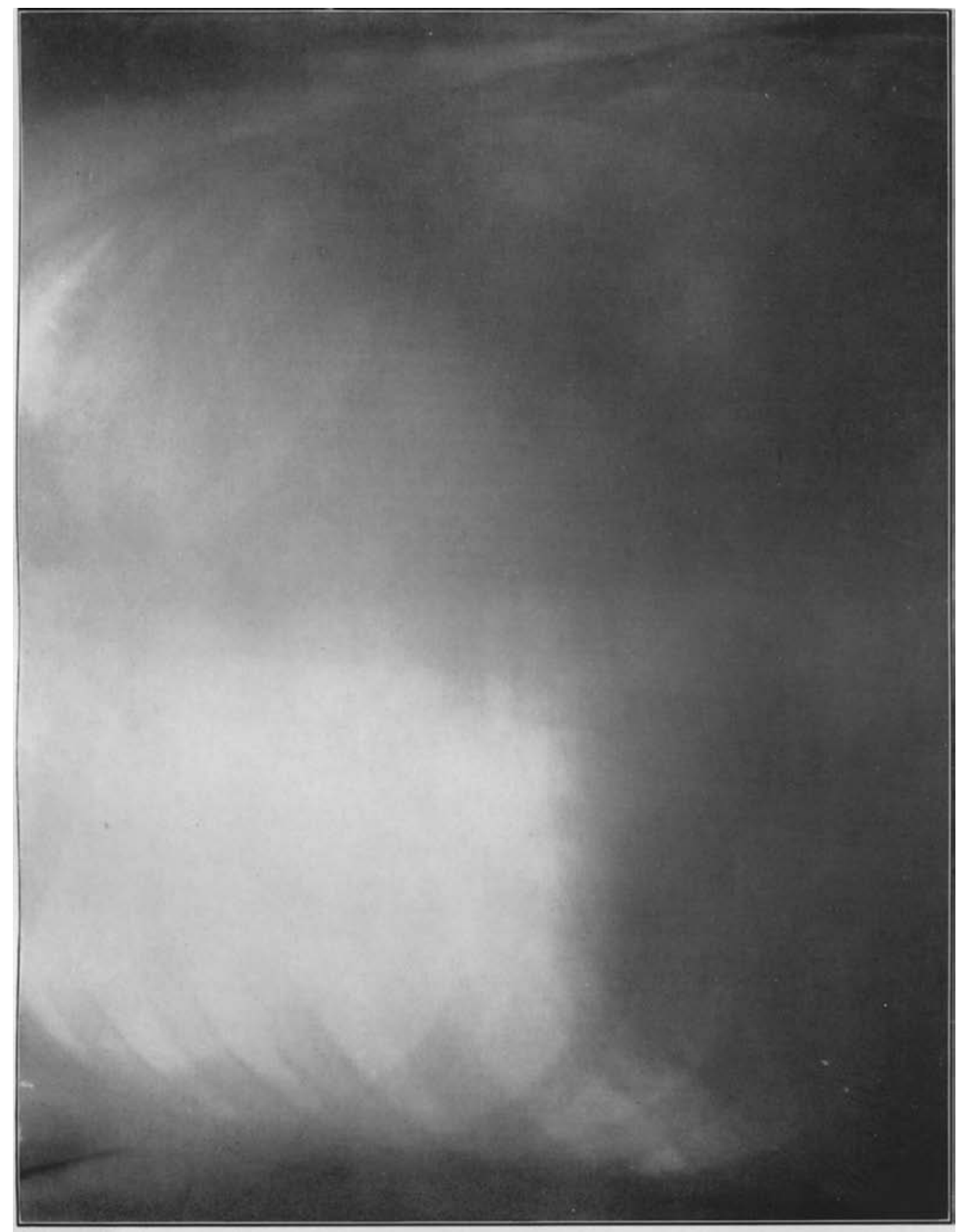




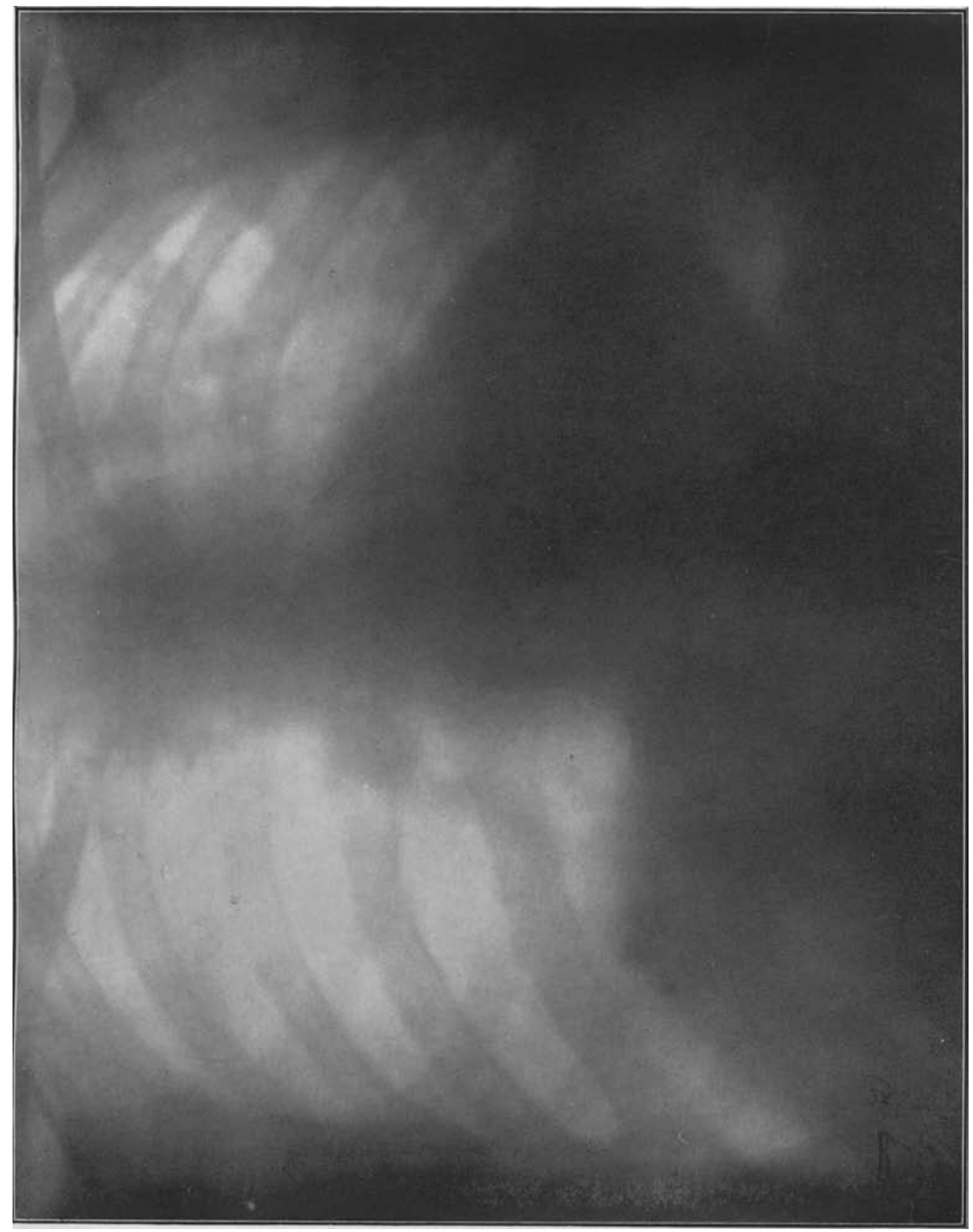




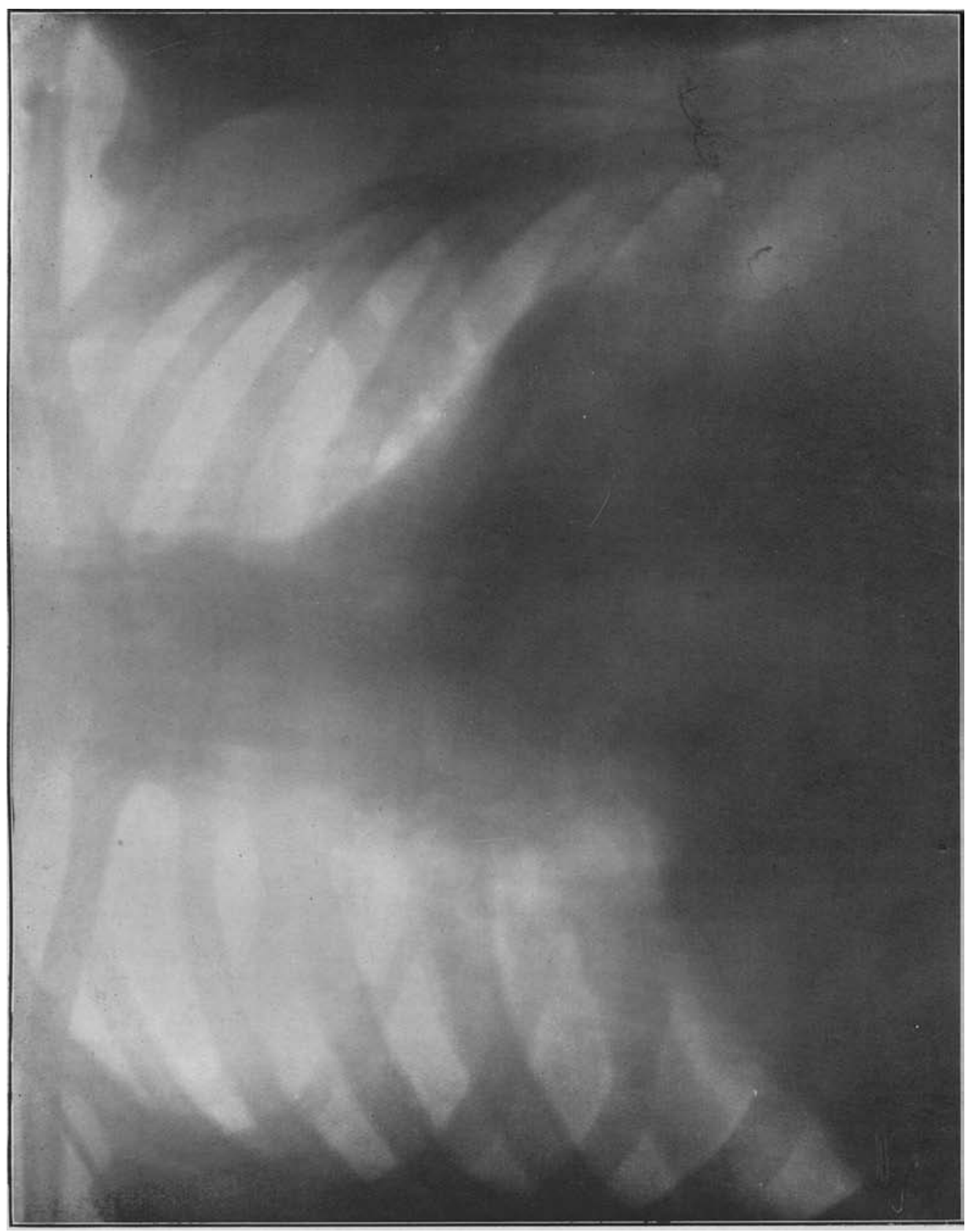

